\title{
Predictors of antiproliferative effect of lanreotide autogel in advanced gastroenteropancreatic neuroendocrine neoplasms
}

\author{
Faidon-Marios Laskaratos ${ }^{1}$ - Eleni Armeni ${ }^{1} \cdot$ Heer Shah $^{2} \cdot$ Maria Megapanou $^{1} \cdot$ Dimitrios Papantoniou $^{1}$. \\ Aimee R Hayes ${ }^{1}$ - Shaunak Navalkissoor ${ }^{3}$. Gopinath Gnanasegaran ${ }^{3}$. Conrad von Stempel ${ }^{4} \cdot$ Edward Phillips $^{1}$. \\ Myles Furnace $^{1} \cdot$ Lukasz Kamieniarz $^{2} \cdot$ Margarita Kousteni $^{2} \cdot$ Tu Vinh Luong $^{5} \cdot$ Jennifer Watkins ${ }^{5}$. \\ Dalvinder Mandair ${ }^{1} \cdot$ Martyn Caplin $^{1} \cdot$ Christos Toumpanakis $^{1}$
}

Received: 30 July 2019 / Accepted: 27 August 2019 / Published online: 25 September 2019

(c) The Author(s) 2019

\begin{abstract}
Purpose The antiproliferative properties of lanreotide autogel (LAN) in gastroenteropancreatic neuroendocrine neoplasms (GEP NENs) were demonstrated in the CLARINET study. However, there is limited literature regarding factors that affect progression-free survival (PFS) in patients with GEP NENs treated with LAN.

Methods We identified a total of 191 treatment-naive patients with advanced GEP NENs and positive SSTR uptake on imaging (Octreoscan or ${ }^{68}$ Gallium DOTATATE Positron Emission Tomography [ $\left.{ }^{68} \mathrm{GaPET}\right]$ ) who received first-line LAN monotherapy, albeit at various starting doses $(60,90$ or $120 \mathrm{mg} / \mathrm{month})$. A group of 102 patients who initiated treatment at the standard dose of $120 \mathrm{mg} / \mathrm{month}$ were included in the study and further evaluated by univariate and multivariate analyses to identify predictors of PFS. Results The location of tumour primary was in the small bowel in $63(62 \%)$, pancreas in 31 (30\%) and colon/rectum in 8 patients $(8 \%)$. The tumours were well-differentiated, and the majority were grade $1(52 \%)$, or $2(38 \%)$. About $60 \%$ of cases had progressive disease at the time of treatment initiation. Most patients with available pretreatment nuclear medicine imaging (Octreoscan or ${ }^{68} \mathrm{Ga}$ PET) had a Krenning score of $3(44 \%)$ or $4(50 \%)$. The median PFS for the entire cohort was 19 months (95\% CI 12, 26 months). The univariate analysis demonstrated that grade 2 tumours, progressive disease at baseline and metastatic liver disease were associated with a significantly shorter PFS, while other evaluated variables did not affect PFS at a statistically significant level. However, at multivariate analysis only the tumour grade remained statistically significant. Conclusions The current study showed that, of many evaluated variables, only the tumour grade was predictive of PFS duration and this should be considered during patient selection for treatment.
\end{abstract}

Keywords Lanreotide autogel $\cdot$ Neuroendocrine tumour $\cdot$ Somatostatin analogue $\cdot$ Neuroendocrine neoplasm

\section{List of abbreviations}

CgA Chromogranin A

CHD Carcinoid heart disease

CI Confidence interval

GEP Gastroenteropancreatic

Supplementary information The online version of this article (https:// doi.org/10.1007/s12020-019-02086-6) contains supplementary material, which is available to authorized users.

Faidon-Marios Laskaratos

flaskaratos@gmail.com

1 Centre for Gastroenterology, Neuroendocrine Tumour Unit, ENETS Centre of Excellence, Royal Free London NHS Foundation Trust, London, UK

2 UCL Medical School, London, UK

$\begin{array}{ll}\text { 5-HIAA } & \text { 5-hydroxyindoleacetic acid } \\ \text { HR } & \text { Hazard ratio } \\ \text { LAN } & \text { Lanreotide autogel } \\ \text { NEC } & \text { Neuroendocrine carcinoma } \\ \text { NEN } & \text { Neuroendocrine neoplasm } \\ \text { NET } & \text { Neuroendocrine tumour } \\ \text { PFS } & \text { Progression-free survival } \\ \text { SSA } & \text { Somatostatin analogue }\end{array}$

3 Department of Nuclear Medicine, Royal Free London NHS Foundation Trust, London, UK

4 Department of Radiology, Royal Free London NHS Foundation Trust, London, UK

5 Academic Centre for Cellular Pathology, Royal Free London NHS Foundation Trust, London, UK 


\section{Introduction}

Neuroendocrine neoplasms (NENs) (term encompassing both well-differentiated neuroendocrine tumours [NETs] and poorly differentiated neuroendocrine carcinomas) are rare neoplasms arising from cells of the diffuse endocrine system. Although they share some common features, there is significant heterogeneity in their clinical presentation, behavioural characteristics and natural history $[1,2]$.

Somatostatin analogues (SSAs) are synthetic derivatives of human somatostatin and are considered the mainstay of therapy for both symptomatic management and tumour growth. The antiproliferative role of long-acting SSAs was confirmed in the PROMID [3] and CLARINET [4] studies. The CLARINET trial demonstrated the antiproliferative effects of lanreotide autogel (LAN) in patients with metastatic, well- or moderately differentiated, non-functional enteropancreatic NETs [4]. An open label extension of the CLARINET study provided additional information regarding the antiproliferative effects of LAN, which was associated with a median progression-free survival (PFS) of 30.8 months. In addition, patients in the placebo group with progressive disease who then switched to open label LAN had a median PFS of 14 months (95\% CI 10.1, not reached) [5].

Currently there is limited literature on factors that predict PFS in patients with NENs receiving SSAs. A recent study from our centre focused on predictive factors affecting PFS in patients with advanced NENs treated with octreotide LAR and identified several predictors of response [6]. The present study aimed to determine the median PFS and evaluate factors affecting PFS in a cohort of patients with gastroenteropancreatic (GEP) NENs treated with LAN $120 \mathrm{mg} / \mathrm{month}$ as first-line monotherapy in a tertiary centre.

\section{Materials and methods}

\section{Patients}

A total of 191 patients were initially identified in our database who had well-differentiated GEP NENs and received first-line LAN monotherapy, albeit at different starting dosing regimens. All cases had a confirmed histopathological diagnosis and were treated in our centre between 2000 and 2016. These patients were treatmentnaive and had nuclear medicine imaging studies confirming positive SSTR uptake (Octreoscan or ${ }^{68} \mathrm{Ga}-\mathrm{PET}$ ) prior to commencing therapy. LAN injections were administered at the standard dose of $120 \mathrm{mg} / 28$ days in 102 patients $(53 \%$ of cases), who were included in the study and evaluated further by univariate and multivariate analyses to identify predictors of response to therapy.
Some of the LAN-treated GEP NET patients had been started on LAN before publication of the CLARINET study data and therefore lower starting doses were used (either $60 \mathrm{mg} / 28$ days [18\%] or $90 \mathrm{mg} / 28$ days [29\%]). These patients were excluded from further analyses to allow the study of a more homogeneous patient cohort that received the same starting dose of LAN.

\section{Study design}

A retrospective review of electronic patient records was performed. Data collected comprised patient demographics (age, gender) and other baseline information (medical comorbidities, treatment indication for SSA), tumour characteristics (location of primary, primary resection, functional status, grade, presence of carcinoid heart disease [CHD], presence of liver or extrahepatic metastases, SSTR uptake on Octreoscan or ${ }^{68} \mathrm{Ga}$ PET imaging and PFS), as well as biochemical data at baseline (serum chromogranin A [CgA] and 5-hydroxyindoleacetic acid [5-HIAA] levels prior to SSA therapy). Patients with active secondary malignancy were excluded from the study.

NETs were classified and graded according to the WHO 2019 classification [7]. The extent of liver involvement was assessed using the following scoring system: (0) absence of liver metastases, (1) volume of liver metastases affecting $<25 \%$ of hepatic parenchyma, (2) volume of liver metastases affecting $25-50 \%$ of hepatic parenchyma and (3) volume of liver disease $>50 \%$.

SSTR uptake on nuclear medicine imaging studies (Octreoscan or ${ }^{68} \mathrm{Ga}$ PET imaging) was classified according to the Krenning scale (1) uptake less than background liver, (2) uptake equal to background liver, (3) uptake greater than background liver, (4) uptake greater than spleen. SSTR uptake was evaluated using Octreoscan or ${ }^{68} \mathrm{Ga}$ PET and pretreatment nuclear medicine studies were available for review in 76\% (78/102) of patients. Baseline (pretreatment) Octreoscan was not available for evaluation in $24 \%$ of cases, usually because it was performed at another institution. However, this had been reviewed prior to LAN commencement in our multidisciplinary tumour board and had demonstrated positive SSTR uptake. Thus, all the included patients had positive SSTR-uptake status before treatment, but the Krenning score could only be precisely evaluated in $76 \%$ of cases. Although the Krenning score was originally used and validated for Octreoscan, a common approach for characterising uptake on ${ }^{68} \mathrm{Ga}$-PET is to use a 'modified' Krenning score, where the same qualitative approach (comparison with the liver uptake) is applied to SSTR-PET imaging $[8,9]$.

Radiological assessment was based on cross-sectional imaging (CT or MRI) in 4-6 month intervals, using the revised RECIST (version 1.1) criteria to determine disease 
progression [10]. A number of variables were assessed as potential predictive factors of response to treatment with LAN. These included:

1. Demographic data: age $(<65$ or $\geq 65)$, sex (male or female) and comorbidity ( $<2$ or $\geq 2$ medical comorbidities).

2. Tumour-related characteristics: primary site (small intestinal, pancreatic or colorectal), tumour grade (1 or 2 using the WHO 2019 classification; G3 and poorly differentiated neoplasms were excluded from the study), functionality (presence or not of functional symptoms) and extent of metastatic spread, including both liver disease burden (none, $<25 \%$, $25-50 \%$ or $>50 \%)$ and extrahepatic sites $(<2$ or $\geq 2)$, which were also evaluated separately as predictors of response (i.e., presence of skeletal, peritoneal, lung, breast and lymph node metastases above and below the diaphragm). In addition, we evaluated SSTR uptake (using the Krenning scale), disease status (stable or progressive disease; progressive status at baseline was defined as disease progression during the previous 12 months before LAN initiation) and association with CHD (for small intestinal NENs).

3. Biomarker levels: $\mathrm{CgA}$ and urinary 5-HIAA (for small intestinal NENs) (using the following categories: normal levels, $<5$ times, $5-10$ times or $>10$ times the upper limit of the normal range).

Their relevance as predictors of response to LAN therapy was assessed by univariate and multivariate analyses.

Tumour biomarker levels (CgA and urinary 5-HIAA for small intestinal NETs) before (within 3 months of) treatment initiation and 12 months after starting LAN were also recorded to monitor biochemical response to therapy.

\section{Statistical analysis}

Non-parametric Kaplan-Meier techniques were used to evaluate PFS, including the median and associated 95\% confidence intervals, in strata defined by various explanatory variables. PFS was defined as the period from the initiation of treatment (LAN $120 \mathrm{mg} / \mathrm{monthly}$ ) until disease progression or death. A semi-parametric Cox regression model was used to perform multivariate analysis, using variables identified as statistically significant by univariate analysis. A $p$-value $<0.05$ was considered statistically significant. Statistical analysis was performed using SPSS version 25 .

\section{Results}

\section{Patient characteristics}

A total of 102 patients were included in this analysis. The mean age $( \pm \mathrm{SD})$ of the patient population was $62 \pm 12$ years. The male to female ratio in the study was $1.2: 1$. The location of tumour primary was in the small bowel in 63 $(62 \%)$, pancreas in $31(30 \%)$ and colon/rectum in 8 patients (8\%). The histological grade was G1 in $53(52 \%)$, G2 in 39 (38\%) and not available in 10 patients $(10 \%)$. The study population included 17 patients (17\%) with no liver disease, 66 patients $(65 \%)$ with liver disease volume $<25 \%, 12$ patients $(12 \%)$ with $25-50 \%$ liver tumour burden and 7 (7\%) with a liver disease volume $>50 \%$. The primary tumour was resected before commencement of LAN in 53 patients (52\%), all of whom had residual disease and were commenced on SSA therapy after surgery. The commonest sites of extrahepatic disease were the bones (present in 18\% of cases), lymph nodes above (13\%) and below the diaphragm $(56 \%)$, peritoneum $(28 \%)$, followed by the lung $(5 \%)$ and breast $(1 \%)$. Indications for commencement of LAN included functional symptoms in $38(37 \%)$ and radiological progression in $61(60 \%)$. Of these patients, 20 had a combination of functional symptoms and radiological progression. There were also 17 patients $(17 \%)$ who were asymptomatic with stable disease and initiated on treatment based on the antiproliferative effects of SSAs. The patients in the latter group were diagnosed after 2014, when data from the CLARINET study emerged and supported the antiproliferative effects of LAN in non-functioning GEP NETs with stable disease status. The indication for treatment initiation was not clearly documented in six cases. Nuclear medicine studies prior to initiation of SSA treatment were available for review in $76 \%$ of cases (78/102). SSTR uptake was evaluated using Octreoscan in 45\% (35/ 78 ) and 68Ga PET in 55\% (43/78) of those cases. Most evaluated patients had a Krenning score of $3(44 \%)$ or $4(50 \%)$.

A summary of patient baseline characteristics is provided in Table 1.

\section{Progression-free survival}

The median PFS for the entire cohort was 19 months (95\% CI 12, 26 months) (Fig. 1).

\section{Univariate analysis of factors influencing progression-free survival}

The univariate analysis demonstrated that grade 2 tumours, progressive disease at initiation of treatment and metastatic 
Table 1 Summary of baseline characteristics in a cohort of 102 patients with advanced low and intermediate grade neuroendocrine tumours treated with standard doses of lanreotide autogel as first-line monotherapy

\begin{tabular}{|c|c|}
\hline & $N=102(\%)$ \\
\hline \multicolumn{2}{|l|}{ Sex } \\
\hline Male & $55(54)$ \\
\hline Female & $47(46)$ \\
\hline \multicolumn{2}{|l|}{ Indication for treatment } \\
\hline Functional symptoms & $38(37)$ \\
\hline Radiological progression & $61(60)$ \\
\hline Asymptomatic and stable disease & $17(17)$ \\
\hline Not clearly documented & $6(6)$ \\
\hline \multicolumn{2}{|l|}{ Location of primary } \\
\hline Small bowel & $63(62)$ \\
\hline Pancreas & $31(30)$ \\
\hline Colon \& Rectum & $8(8)$ \\
\hline \multicolumn{2}{|l|}{ Grade } \\
\hline G1 & $53(52)$ \\
\hline G2 & $39(38)$ \\
\hline Unknown & $10(10)$ \\
\hline \multicolumn{2}{|l|}{ Functional Status } \\
\hline Functional & $38(37)$ \\
\hline Non-functional & $64(63)$ \\
\hline \multicolumn{2}{|l|}{ Carcinoid heart disease } \\
\hline Yes & $7(7)$ \\
\hline No & $95(93)$ \\
\hline \multicolumn{2}{|l|}{ Chromogranin A } \\
\hline Normal & $17(17)$ \\
\hline Up to 5 times higher than upper limit of normal & $13(13)$ \\
\hline $\begin{array}{l}\text { Between 5-10 times higher than upper limit } \\
\text { of normal }\end{array}$ & $13(13)$ \\
\hline$>10$ times upper limit of normal & $16(16)$ \\
\hline Unknown & $43(42)$ \\
\hline \multicolumn{2}{|l|}{ Urinary 5-HIAA } \\
\hline Normal & $19(19)$ \\
\hline Up to 5 times higher than upper limit of normal & $11(11)$ \\
\hline $\begin{array}{l}\text { Between 5-10 times higher than upper limit } \\
\text { of normal }\end{array}$ & $4(4)$ \\
\hline$>10$ times upper limit of normal & $11(11)$ \\
\hline Unknown & $57(56)$ \\
\hline \multicolumn{2}{|l|}{ Primary tumour resection } \\
\hline Yes & $53(52)$ \\
\hline No & $49(48)$ \\
\hline \multicolumn{2}{|l|}{ Krenning Score } \\
\hline Not available & $24(24)$ \\
\hline 1 & $0(0)$ \\
\hline 2 & $5(5)$ \\
\hline 3 & $34(33)$ \\
\hline 4 & $39(38)$ \\
\hline
\end{tabular}

liver disease were predictive factors of a significantly shorter PFS.

\section{Tumour grade}

The median PFS was 24 months (95\% CI: 11, 37 months) for patients with G1 tumours, while patients with G2 tumours had a median PFS of 12 months (95\% CI: 6, 18 months) (Fig. 2).

\section{Disease status at initiation of treatment}

The median PFS was 31 months (95\% CI: 21, 41) in patients with stable disease, while patients with progressive disease had a median PFS of 13 months (95\% CI: 9, 17) (Fig. 3).

\section{Liver involvement}

The median PFS was not reached for patients without liver involvement, while it was 12 months (95\% CI: 8, 16 months) for those with liver disease <25\%, 21 months (95\% CI: 0, 49 months) for those with liver tumour burden 25-50\%, and 34 months (95\% CI: 11, 60 months) for patients with liver disease $>50 \%$ (Fig. 4).

In contrast, age, gender, primary tumour site, presence of $\mathrm{CHD}$, other metastases, resection of the tumour primary, SSTR uptake, baseline $\mathrm{CgA}$ and urinary 5-HIAA levels were not found to be predictive factors affecting response to treatment.

The results of the univariate analysis are shown in Table 2 .

\section{Multivariate analysis of factors influencing progression-free survival}

The multivariate analysis demonstrated that grade 2 tumours (HR 1.64, 95\% CI: 1.01, 2.67, $p=0.04$ ) remained an independent predictor of shorter PFS, while the presence of progressive disease and liver disease volume did not retain statistical significance. The results of the multivariate analysis are shown in Table 3.

\section{Tumour biomarker responses}

CgA levels were available for evaluation at baseline and 12 months after LAN initiation in 49 cases (48\% of study population). $\mathrm{CgA}$ levels decreased by $54 \%$ on average and 26 of these patients $(53 \%)$ had a significant $(>50 \%)$ drop in their CgA levels.

In addition, urinary 5-HIAA levels were available for assessment at baseline and 12 months after LAN initiation in 12 patients (19\%) with SINENs. Urinary 5-HIAA levels 
Fig. 1 Kaplan-Meier estimate of the survival function for the time to tumour progression for the entire cohort of 102 patients. The median PFS was 19 months (95\% CI 12, 26 months)
Fig. 2 Kaplan-Meier estimate of the survival function for the PFS in patients with advanced NETs receiving lanreotide autogel, stratified by tumour grade at diagnosis
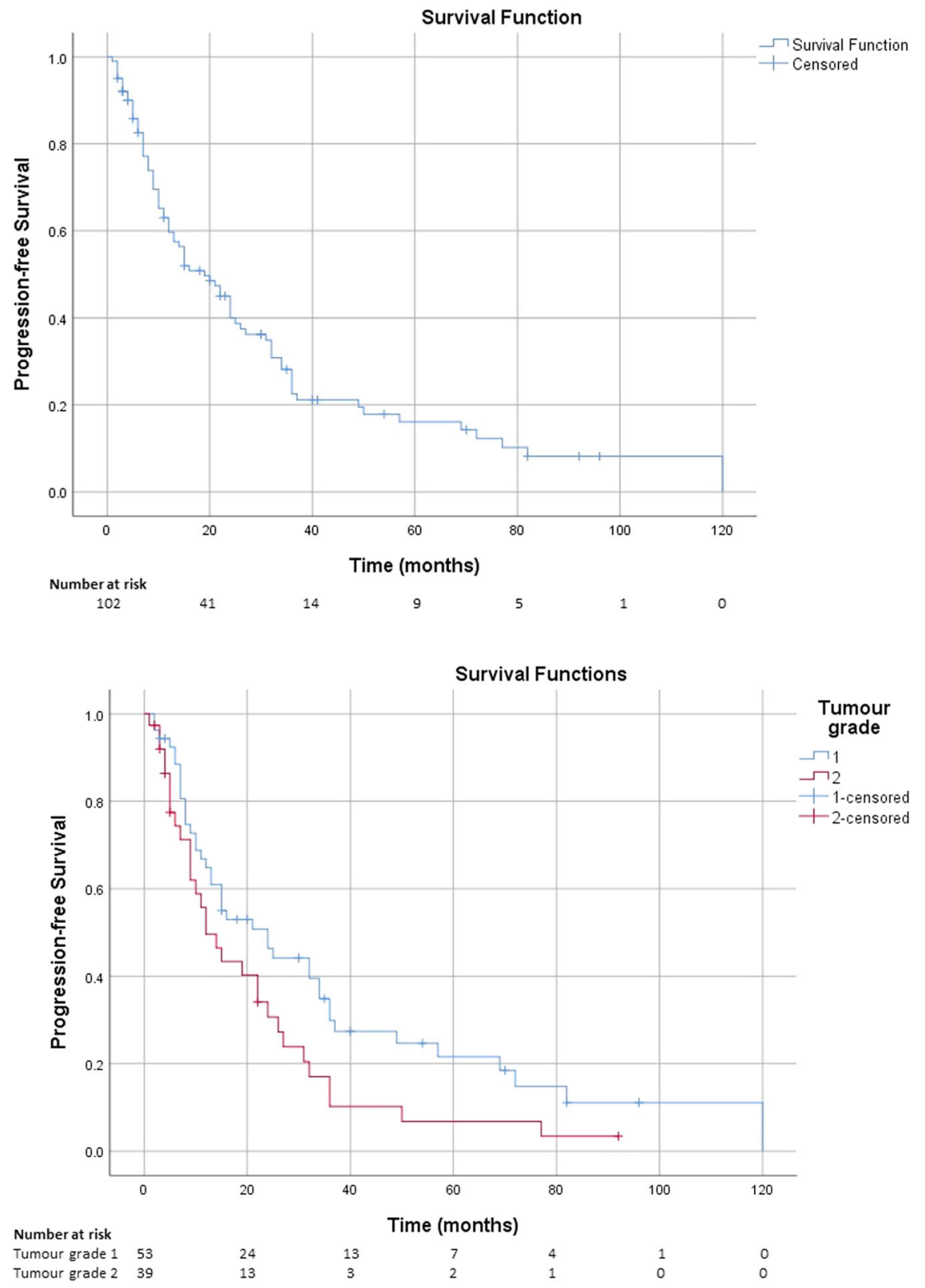

decreased by an average of $36 \%$ and six of these patients $(50 \%)$ had a significant $(>50 \%)$ drop in their urinary 5HIAA levels.

\section{Discussion}

The present study showed that the median PFS in a large institutional cohort of 102 treatment-naive patients with low/intermediate grade GEP NETs treated with first-line standard dose LAN monotherapy was 19 months. In the univariate analysis the response to treatment was adversely affected by hepatic tumour load, progressive disease status and tumour grade (grade 2) at initiation of treatment.
However, at multivariate analysis only the tumour grade was independently associated with PFS duration. Other factors (age and gender, location of primary tumour, surgical resection of primary tumour, presence of extrahepatic metastases, SSTR uptake, baseline levels of CgA or urinary 5-HIAA) did not appear to be predictive of response to treatment. In a previous large retrospective study from our centre evaluating predictors of PFS in a cohort of 254 treatment naive patients with NET treated with octreotide LAR, the median PFS was 37 months and liver tumour burden, higher tumour grade (grade 2) and pancreatic primaries were predictive of a shorter PFS [6].

Other studies that evaluated the median PFS in patients on LAN treatment for the management of GEP or bronchial 
Fig. 3 Kaplan-Meier estimate of in patients with advanced NETs receiving lanreotide autogel, stratified by disease status (progressive vs. stable) at initiation of treatment the survival function for the PFS

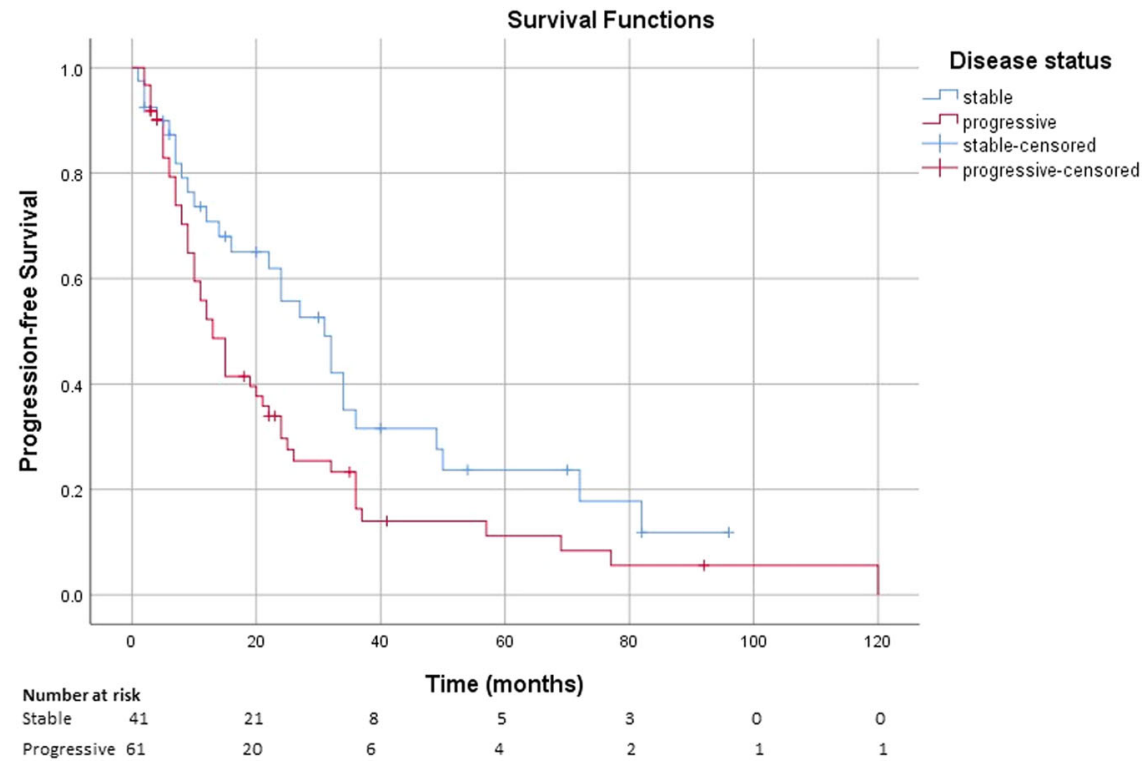

Fig. 4 Kaplan-Meier estimate of the survival function for the PFS in patients with advanced NETs receiving lanreotide autogel, stratified by $\%$ liver disease volume at diagnosis

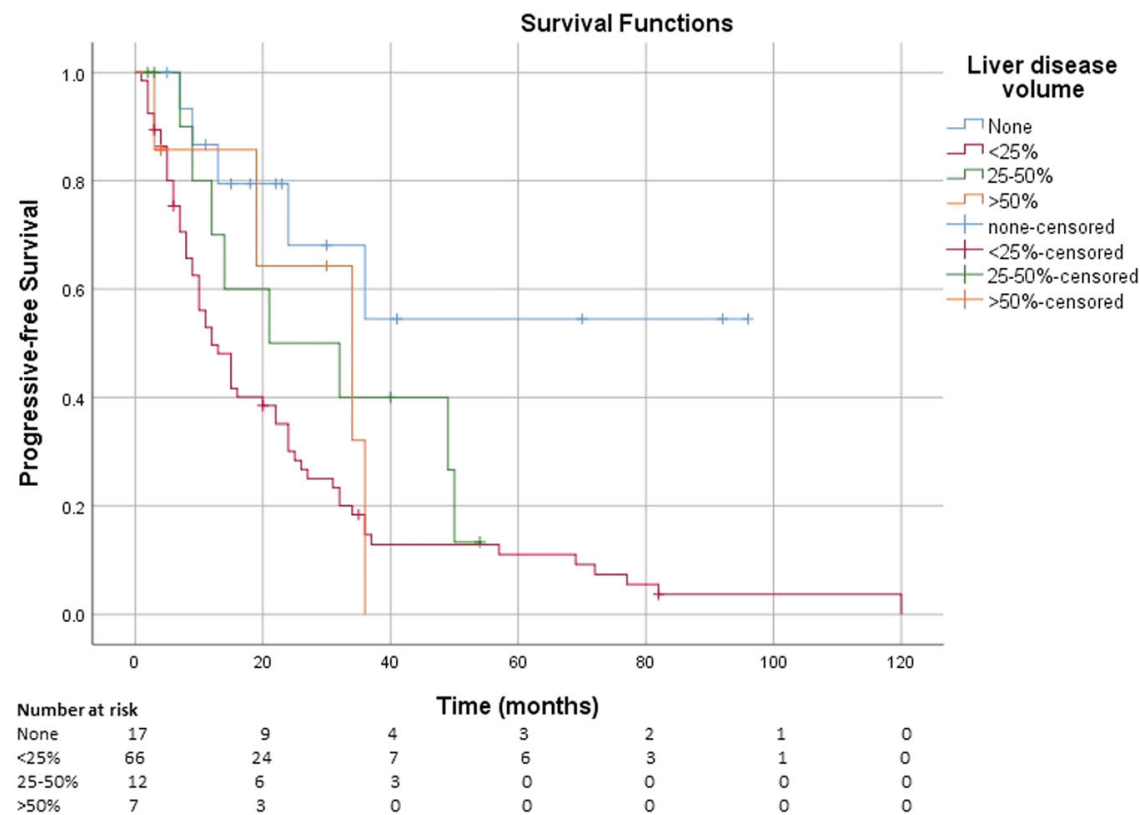

NETs are summarised in Supplementary File 1 (Table 1a, b) $[4,5,11-21]$. The PFS reported in our study is in agreement with other publications $[4,5,13,14,16,20,21]$. The CLARINET and CLARINET open label extension study demonstrated the efficacy of LAN monotherapy as a treatment modality of patients with GEP NETs of grade 1 or 2 (with Ki67<10\%) [4, 5]. However, there is limited literature regarding predictors of LAN efficacy.

The present study demonstrated that tumour grade was the only predictive factor independently associated with PFS duration. G2 tumours were associated with a significantly shorter PFS and an HR of 1.64 (95\% CI: 1.01, 2.67) $(p=0.04)$. In agreement with our findings, a Spanish multicentre study confirmed that lower Ki67 ranking was associated with a lower risk of disease progression (Ki67 ranking, HR: $1.17,95 \% \mathrm{CI}: 1.03,1.33 ; p$-value $=0.02$ ) [11]. Similarly, in another study patients with Ki67 $\geq 5 \%$ demonstrated an up to $73.8 \%$ higher risk of disease progression on LAN treatment compared with patients diagnosed with $\mathrm{Ki} 67<5 \%$ at baseline [13].

In addition, our study showed an association between progressive disease status at initiation of LAN treatment and risk of further disease progression at univariate analysis, but this factor was no longer significant at the multivariate analysis. The median PFS of patients with progressive disease at baseline was 13 months (95\% CI: 9, 17), while those patients with stable disease had a median PFS was 31 months (95\% CI: 21, 41). These findings are in keeping 
Table 2 Univariate analysis of predictive factors of response to standard doses of first-line lanreotide autogel in a cohort of 102 advanced low and intermediate grade neuroendocrine tumours

\begin{tabular}{|c|c|c|c|c|c|}
\hline Variable & Category & HR & 95\% CI (lower) & 95\% CI (upper) & $p$-value \\
\hline \multirow[t]{2}{*}{ Age } & $<65$ & (ref) & & & \\
\hline & $\geq 65$ & 1.28 & 0.81 & 2.02 & 0.29 \\
\hline \multirow[t]{2}{*}{ Sex } & Male & (ref) & & & \\
\hline & Female & 0.87 & 0.57 & 1.41 & 0.64 \\
\hline \multirow[t]{2}{*}{ Medical comorbidities } & $<2$ & (ref) & & & \\
\hline & $\geq 2$ & 1.31 & 0.72 & 2.40 & 0.38 \\
\hline \multirow[t]{3}{*}{ Tumour primary } & Small bowel & (ref) & & & \\
\hline & Pancreas & 0.91 & 0.54 & 1.53 & 0.72 \\
\hline & Colon and rectum & 1.53 & 0.72 & 3.25 & 0.27 \\
\hline \multirow[t]{2}{*}{ Tumour grade } & G1 & (ref) & & & \\
\hline & G2 & 1.60 & 0.99 & 2.57 & $0.049^{\mathrm{a}}$ \\
\hline \multirow[t]{3}{*}{ Krenning score } & 2 & (ref) & & & \\
\hline & 3 & 0.39 & 0.09 & 1.74 & 0.22 \\
\hline & 4 & 0.39 & 0.09 & 1.72 & 0.21 \\
\hline \multirow[t]{2}{*}{ Primary tumour resection } & No & (ref) & & & \\
\hline & Yes & 1.20 & 0.75 & 1.91 & 0.44 \\
\hline \multirow[t]{4}{*}{ Liver disease volume } & None & (ref) & & & \\
\hline & $<25 \%$ & 4.19 & 1.68 & 10.46 & $0.002^{\mathrm{a}}$ \\
\hline & $25-50 \%$ & 2.56 & 0.83 & 7.85 & 0.10 \\
\hline & $>50 \%$ & 2.79 & 0.75 & 10.48 & 0.13 \\
\hline \multirow[t]{2}{*}{ Tumour functionality } & No & (ref) & & & \\
\hline & Yes & 1.49 & 0.94 & 2.35 & 0.09 \\
\hline \multirow{2}{*}{ Disease status at initiation of treatment } & Stable & (ref) & & & \\
\hline & Progressive & 1.64 & 1.02 & 2.64 & $0.04^{\mathrm{a}}$ \\
\hline \multirow[t]{2}{*}{ Extrahepatic metastases } & $<2$ sites & (ref) & & & \\
\hline & $\geq 2$ sites & 1.07 & 0.67 & 1.70 & 0.78 \\
\hline \multirow[t]{2}{*}{ Lymphadenopathy above the diaphragm } & No & $(\mathrm{ref})$ & & & \\
\hline & Yes & 0.69 & 0.33 & 1.44 & 0.32 \\
\hline \multirow[t]{2}{*}{ Lymphadenopathy below the diaphragm } & No & (ref) & & & \\
\hline & Yes & 0.83 & 0.52 & 1.31 & 0.42 \\
\hline \multirow[t]{2}{*}{ Skeletal metastases } & No & $(\mathrm{ref})$ & & & \\
\hline & Yes & 1.48 & 0.81 & 2.70 & 0.20 \\
\hline \multirow[t]{2}{*}{ Lung metastases } & No & (ref) & & & \\
\hline & Yes & 1.49 & 0.54 & 4.10 & 0.44 \\
\hline \multirow[t]{2}{*}{ Peritoneal metastases } & No & (ref) & & & \\
\hline & Yes & 0.74 & 0.45 & 1.22 & 0.24 \\
\hline \multirow[t]{2}{*}{ Breast metastases } & No & $(\mathrm{ref})$ & & & \\
\hline & Yes & 0.70 & 0.10 & 5.03 & 0.72 \\
\hline \multirow[t]{2}{*}{ Carcinoid heart disease $^{\mathrm{b}}$} & No & (ref) & & & \\
\hline & Yes & 0.41 & 0.13 & 1.31 & 0.13 \\
\hline \multirow[t]{4}{*}{ Chromogranin A levels } & Normal & (ref) & & & \\
\hline & $<5 \times$ the upper limit of normal & 0.97 & 0.43 & 2.17 & 0.93 \\
\hline & $5-10 \times$ the upper limit of normal & 0.76 & 0.33 & 1.74 & 0.52 \\
\hline & $>10 \times$ the upper limit of normal & 0.65 & 0.28 & 1.49 & 0.31 \\
\hline \multirow[t]{4}{*}{ Urinary 5-HIAA levels $^{\mathrm{b}}$} & Normal & (ref) & & & \\
\hline & $<5 \times$ the upper limit of normal & 0.58 & 0.23 & 1.44 & 0.24 \\
\hline & $5-10 \times$ the upper limit of normal & 1.23 & 0.41 & 3.75 & 0.71 \\
\hline & $>10 \times$ the upper limit of normal & 0.36 & 0.12 & 1.14 & 0.08 \\
\hline
\end{tabular}

${ }^{a}$ Denotes statistical significance $(p<0.05)$

${ }^{\mathrm{b}}$ Analysis restricted to small bowel NETs

with the CLARINET-OLE study, in which patients with progressive disease who received LAN had a median time to further progression of 14 months [5], and this was significantly shorter than the reported PFS for patients with stable disease, suggesting an inverse association between LAN efficacy and disease progression at initiation of 
Table 3 Multivariate analysis of predictors of response to first-line monotherapy with standard doses of lanreotide autogel in a cohort of 102 patients with advanced low/intermediate grade neuroendocrine tumours

\begin{tabular}{|c|c|c|c|c|c|}
\hline Variable & Category & HR & 95\% CI (lower) & 95\% CI (upper) & $p$-value \\
\hline \multirow[t]{4}{*}{ Liver disease volume } & None & (ref) & & & \\
\hline & $<25 \%$ & 0.39 & 0.10 & 1.48 & 0.17 \\
\hline & $25-50 \%$ & 1.30 & 0.47 & 3.60 & 0.62 \\
\hline & $>50 \%$ & 1.00 & 0.29 & 3.45 & 0.99 \\
\hline \multirow[t]{2}{*}{ Disease status at initiation of treatment } & Stable & (ref) & & & \\
\hline & Progressive & 1.49 & 0.87 & 2.52 & 0.14 \\
\hline \multirow[t]{2}{*}{ Tumour grade } & G1 & (ref) & & & \\
\hline & G2 & 1.64 & 1.01 & 2.67 & $0.04^{*}$ \\
\hline
\end{tabular}

*indicates statistical significance

treatment. However, our analysis did not identify this as an independent factor of LAN efficacy.

Furthermore, the presence of liver metastases was associated with a significantly shorter PFS at univariate analysis, since patients with $<25 \%$ liver disease volume had a significantly shorter PFS compared with patients without liver metastases. However, there was absence of proportionality of the risk of progression in strata defined by the volume of liver disease and this factor was no longer statistically significant by multivariate analysis. Therefore, our study did not confirm the significance of liver tumour burden as an independent risk factor for progression on first-line LAN monotherapy. The predictive role of the hepatic tumour load in the response to SSA treatment has been assessed only in a few studies. In the PROMID trial [3], the only independent prognostic factor for prolonged time to progression or tumour-related death was a low hepatic tumour burden $(\leq 10 \%)$. In the CLARINET trial [4], a shorter PFS was demonstrated in patients with hepatic tumour burden $>25 \%$ although both groups received benefit with LAN. Further studies would be useful to define the impact of liver disease volume on LAN efficacy.

Limitations of this study include its retrospective design and the potential interpretation bias. Another limitation of our study is that the proportion of patients with high hepatic tumour burden at baseline was relatively small, and therefore this subgroup of patients is relatively under-represented. In addition, this study was conducted in a single tertiary referral centre and therefore our clinical practices and patient population may not be typical of those in smaller centres. Furthermore, most cases had small bowel primary tumours, which means that our findings might be less applicable to patients with pancreatic and colorectal NENs, that were smaller subgroups of our study. However, the primary tumour site did not appear to significantly affect PFS, although this may be subject to selection bias. Another limitation of this study is that the Krenning score was evaluated by Octreoscan in some patients and ${ }^{68} \mathrm{Ga}$-PET in others. A recent study analysing head-to-head comparisons of Krenning scores in Octreoscan and ${ }^{68} \mathrm{Ga}$-PET showed that the detection of SSTR-expressing disease (Krenning score $\geq 2$ ) was significantly higher in ${ }^{68} \mathrm{Ga}$-PET compared with Octreoscan. In addition, the Krenning scores were higher in ${ }^{68} \mathrm{Ga}$-PET than in Octreoscan, but this was particularly an issue with small lesions $(<2 \mathrm{~cm})$, while Krenning scores were nearly equivalent with lesions greater than $5 \mathrm{~cm}$ [9]. Although this publication highlights differences in Krenning score assessments using these different imaging modalities, these were more prominent in small lesions. Our study included only patients with advanced (metastatic) disease and therefore most patients had larger lesions that would presumably eliminate many of these differences. However, this is certainly a limitation of this retrospective analysis.

In conclusion, our study population represents the largest cohort of patients in the literature with GEP NENs treated with LAN first-line monotherapy in a single institution and has provided additional information regarding the antiproliferative effects of LAN. Tumour grade appeared to be the only predictive factor independently associated with PFS duration in this analysis. Of course, the management of patients should be discussed within a multidisciplinary tumour board considering the specific characteristics of each individual case. Treatment naive patients with grade 2 GEP NETs selected for treatment with first-line long-acting SSAs should be monitored more closely to identify disease progression and initiate, if necessary, second-line therapeutic strategies at an early stage.

\section{Data availability}

All data generated or analysed in this study are included in this article [and its supplementary information files].

Funding This study was funded by the Royal Free Charity Trustees Grant 311. 
Authors' contributions F.L., E.A. and H.S. wrote the initial draft. F.L., H.S., M.M., D.P., A.H., S.N., G.G., C.S., E.P., M.F., L.K., M.K., T.V. L., J.W. contributed to the data collection. FL performed the data analysis. FL, A.H., T.V.L., J.W., D.M., M.C. and C.T. contributed to the revision of the paper.

\section{Compliance with ethical standards}

Conflict of interest M.C. and C.T. have received from IPSEN honoraria for lectures, advisory boards and educational grants for the Royal Free NET Unit.

Ethical approval This was a retrospective cohort study and formal ethical approval was not required. However, patient data were handled with strict confidentiality and adherence to clinical governance rules in line with our institutional policies.

Publisher's note Springer Nature remains neutral with regard to jurisdictional claims in published maps and institutional affiliations.

Open Access This article is distributed under the terms of the Creative Commons Attribution 4.0 International License (http://crea tivecommons.org/licenses/by/4.0/), which permits unrestricted use, distribution, and reproduction in any medium, provided you give appropriate credit to the original author(s) and the source, provide a link to the Creative Commons license, and indicate if changes were made.

\section{References}

1. T. Enzler, T. Fojo, Long-acting somatostatin analogues in the treatment of unresectable/metastatic neuroendocrine tumors. Semin Oncol. 44(2), 141-156 (2017). https://doi.org/10.1053/ jseminoncol201707001. [Epub $25 \mathrm{Jul}$ 2017]

2. M. Michael, R. Garcia-Carbonero, M.M. Weber, C. LombardBohas, C. Toumpanakis, R.J. Hicks, The antiproliferative role of lanreotide in controlling growth of neuroendocrine tumors: a systematic review. Oncologist 22(3), 272-285 (2017). https://doi. org/10.1634/theoncologist2016-030. [Epub 20 Feb 2017]

3. A. Rinke, H.H. Muller, C. Schade-Brittinger, K.J. Klose, P. Barth, M. Wied, C. Mayer, B. Aminossadati, U.F. Pape, M. Blaker, et al. Placebo-controlled, double-blind, prospective, randomized study on the effect of octreotide LAR in the control of tumor growth in patients with metastatic neuroendocrine midgut tumors: a report from the PROMID Study Group. J. Clin. Oncol. 27(28), 4656-4663 (2009). https://doi.org/10.1200/JCO2009228510. [Epub 24 Aug 2009]

4. M.E. Caplin, M. Pavel, J.B. Cwikla, A.T. Phan, M. Raderer, E. Sedlackova, G. Cadiot, E.M. Wolin, J. Capdevila, L. Wall, et al. Lanreotide in metastatic enteropancreatic neuroendocrine tumors. N. Engl. J. Med 371(3), 224-233 (2014). https://doi.org/10.1056/ NEJMoa1316158

5. M.E. Caplin, M. Pavel, J.B. Cwikla, A.T. Phan, M. Raderer, E. Sedlackova, G. Cadiot, E.M. Wolin, J. Capdevila, L. Wall, et al. Anti-tumour effects of lanreotide for pancreatic and intestinal neuroendocrine tumours: the CLARINET open-label extension study. Endocr. Relat. Cancer 23(3), 191-199 (2016). https://doi. org/10.1530/ERC-15-0490. [Epub 7 Jan 2016]

6. F.M. Laskaratos, M. Walker, K. Naik, E. Maragkoudakis, N. Oikonomopoulos, L. Grant, T. Meyer, M. Caplin, C. Toumpanakis, Predictive factors of antiproliferative activity of octreotide LAR as first-line therapy for advanced neuroendocrine tumours. Br. J. Cancer 115(11), 1321-1327 (2016). https://doi.org/10.1038/bjc2016349. [Epub 3 Nov 2016]
7. World Health Organization, Digestive System Tumours, WHO Classification of Tumours, vol. 1, 5th edn. (World Health Organization, IARC, 150 Cours Albert Thomas, 69372 Lyon CEDEX 08, France, 2019).

8. M.S. Hofman, W.F. Lau, R.J. Hicks, Somatostatin receptor imaging with 68Ga DOTATATE PET/CT: clinical utility, normal patterns, pearls, and pitfalls in interpretation. Radiographics 35(2), 500-516 (2015). https://doi.org/10.1148/rg352140164

9. T.A. Hope, J. Calais, L. Zhang, W. Dieckmann, C. Millo, (111)Inpentetreotide scintigraphy vs. (68)Ga-DOTATATE PET: impact on Krenning scores and effect of tumor burden. J Nucl Med 60(9), 1266-1269 (2019). https://doi.org/10.2967/jnumed.118.223016.

10. E.A. Eisenhauer, P. Therasse, J. Bogaerts, L.H. Schwartz, D. Sargent, R. Ford, J. Dancey, S. Arbuck, S. Gwyther, M. Mooney et al. New response evaluation criteria in solid tumours: revised RECIST guideline (version 1.1). Eur. J. Cancer 45(2), 228-247 (2009). https://doi.org/10.1016/jejca200810026

11. M. Martin-Richard, B. Massuti, E. Pineda, V. Alonso, M. Marmol, D. Castellano, E. Fonseca, A. Galan, M. Llanos, M.A. Sala, et al. Antiproliferative effects of lanreotide autogel in patients with progressive, well-differentiated neuroendocrine tumours: a Spanish, multicentre, open-label, single arm phase II study. BMC Cancer 13(Sep), 427 (2013). https://doi.org/10.1186/1471-2407$13-427$

12. S. Faiss, U.F. Pape, M. Bohmig, Y. Dorffel, U. Mansmann, W. Golder, E.O. Riecken, B. Wiedenmann, Prospective, randomized, multicenter trial on the antiproliferative effect of lanreotide, interferon alfa, and their combination for therapy of metastatic neuroendocrine gastroenteropancreatic tumors-the International Lanreotide and Interferon Alfa Study Group. J. Clin. Oncol. 21 (14), 2689-2696 (2003). https://doi.org/10.1200/JCO200312142

13. M. Palazzo, C. Lombard-Bohas, G. Cadiot, T. Matysiak-Budnik, V. Rebours, M.P. Vullierme, A. Couvelard, O. Hentic, P. Ruszniewski, Ki67 proliferation index, hepatic tumor load, and pretreatment tumor growth predict the antitumoral efficacy of lanreotide in patients with malignant digestive neuroendocrine tumors. Eur. J. Gastroenterol. Hepatol. 25(2), 232-238 (2013). https://doi.org/10.1097/MEG0b013e328359d1a6

14. A. Bianchi, L. De Marinis, A. Fusco, F. Lugli, L. Tartaglione, D. Milardi, M. Mormando, A.P. Lassandro, R. Paragliola, C.A. Rota, et al. The treatment of neuroendocrine tumors with long-acting somatostatin analogs: a single center experience with lanreotide autogel. J. Endocrinol. Investig 34(9), 692-697 (2011). https://doi. org/10.3275/8058. [Epub 7 Nov 2011]

15. M. Ducreux, P. Ruszniewski, J.A. Chayvialle, J. Blumberg, D. Cloarec, H. Michel, J.M. Raymond, J.L. Dupas, H. Gouerou, R. Jian, et al. The antitumoral effect of the long-acting somatostatin analog lanreotide in neuroendocrine tumors. Am. J. Gastroenterol. 95(11), 3276-3281 (2000). https://doi.org/10.1111/j15720241200003210x

16. M.S. Khan, F. El-Khouly, P. Davies, C. Toumpanakis, M.E. Caplin, Long-term results of treatment of malignant carcinoid syndrome with prolonged release Lanreotide (Somatuline Autogel). Aliment Pharmacol. Ther. 34(2), 235-242 (2011). https://doi. org/10.1111/j1365-2036201104693x. [Epub 17 May 2011]

17. J. Capdevila, I. Sevilla, V. Alonso, L. Anton Aparicio, P. Jimenez Fonseca, E. Grande, J.J. Reina, J.L. Manzano, J.D. Alonso Lajara, J. Barriuso, et al. Evaluation of the efficacy and safety of lanreotide in combination with targeted therapies in patients with neuroendocrine tumours in clinical practice: a retrospective crosssectional analysis. BMC Cancer 15(Jul), 495 (2015). https://doi. org/10.1186/s12885-015-1512-6

18. S. Faiss, U. Rath, U. Mansmann, D. Caird, N. Clemens, E.O. Riecken, B. Wiedenmann, Ultra-high-dose lanreotide treatment in patients with metastatic neuroendocrine gastroenteropancreatic 
tumors. Digestion 60(5), 469-476 (1999). https://doi.org/10.1159/ 000007693

19. H. Ida, Y. Honma, H. Hirano, H. Shoji, S. Iwasa, N. Okita, A. Takashima, K. Kato, T. Fukuda, N. Boku, Clinical outcomes of patients with G1/G2 neuroendocrine tumors arising from foregut or hindgut treated with somatostatin analogs: a retrospective study. Investig New Drugs 37(3), 573-578 (2018). https://doi.org/ 10.1007/s10637-018-0669-7

20. F. Panzuto, M. Di Fonzo, E. Iannicelli, R. Sciuto, C.L. Maini, G. Capurso, M. Milione, M.S. Cattaruzza, M. Falconi, V. David, et al. Long-term clinical outcome of somatostatin analogues for treatment of progressive, metastatic, well-differentiated enteropancreatic endocrine carcinoma. Ann. Oncol. 17(3), 461-466 (2006). https://doi.org/10.1093/annonc/mdj113. [Epub 19 Dec 2005]

21. E. Ozaslan, H. Karaca, S. Koca, A. Sevinc, B. Hacioglu, M. Ozkan, M. Ozcelik, A.O. Duran, I. Hacibekiroglu, Y. Yildiz, et al. Comparison of survival with somatostatin analog and chemotherapy and prognostic factors for treatment in 165 advanced neuroendocrine tumor patients with Ki-67 20\% or less. Anticancer Drugs 28(2), 222-229 (2017). https://doi.org/10.1097/ CAD0000000000000445 